\title{
Relationship between Motor Skill Impairments and Motor Imagery Ability in Children with Autism Spectrum Disorders: A Pilot Study Using the Hand Rotation Task
}

\author{
Shogo Hirata ${ }^{*}$, Hideyuki Okuzumi ${ }^{2}$, Yoshio Kitajima ${ }^{3}$, Tomio Hosobuchi ${ }^{4}$, Akio Nakai ${ }^{5}$, \\ Mitsuru Kokubun² \\ ${ }^{1}$ Department of Elementary Education, Ibaraki Christian University, Hitachi, Japan \\ ${ }^{2}$ Faculty of Education, Tokyo Gakugei University, Koganei, Japan \\ ${ }^{3}$ Faculty of Education, Chiba University, Chiba, Japan \\ ${ }^{4}$ Faculty of Education, Saitama University, Saitama, Japan \\ ${ }^{5}$ Hyogo Children's Sleep and Development Medical Research Center, Kobe, Japan \\ Email: ${ }^{*}$ r093002g@st.u-gakugei.ac.jp
}

Received 25 March 2015; accepted 15 May 2015; published 18 May 2015

Copyright (C) 2015 by authors and Scientific Research Publishing Inc.

This work is licensed under the Creative Commons Attribution-NonCommercial International License (CC BY-NC). http://creativecommons.org/licenses/by-nc/4.0/

(c) (i) \& Open Access

\section{Abstract}

The purpose of this study was to examine the relationship between motor skill impairments and motor imagery ability in children with autism spectrum disorders (ASD). The participants were 19 children with ASD, aged 7 - 16 years. We conducted two tasks: movement assessment battery for children-2 (MABC-2) and hand rotation task. The MABC-2 is a motor test that can assess total motor ability, and three sub-domain abilities. Hand rotation is a cognitive task that can evaluate motor imagery ability. Results indicated the possibility that severities of motor skill impairments were interrelated with motor imagery ability in children with ASD. First, fine motor control ability was strongly interrelated with the general response speed of the hand rotation task. Second, balance ability was interrelated with the fit and slope coefficients of the hand rotation task. The internal modeling deficit seems to play an important role in children, either with developmental coordination disorder or ASD.

\section{Keywords}

Autism Spectrum Disorders, Motor Skill Impairments, Motor Imagery, Hand Rotation, MABC-2

"Corresponding author.

How to cite this paper: Hirata, S. et al. (2015). Relationship between Motor Skill Impairments and Motor Imagery Ability in Children with Autism Spectrum Disorders: A Pilot Study Using the Hand Rotation Task. Psychology, 6, 752-759. 


\section{Introduction}

Autism spectrum disorders (ASD) appeared to be biologically-based conditions with an early onset and a lifelong course, and previously included several autistic disorders such as autism, asperger syndrome (AS), and pervasive developmental disorders not otherwise specified (PDD-NOS) in the Diagnostic and Statistical Manual of Mental Disorders 4th Edition text revision (DSM-IV-TR; American Psychiatric Association, 2000). The core features of these disorders are communication and social deficits along with stereotyped behaviours (i.e., American Psychiatric Association, 2013). In recent years, empirical studies have revealed the existence of motor skill impairments in children with ASD, using standardized assessment batteries such as the Movement Assessment Battery for Children (MABC; Henderson \& Sugden, 1992. 2nd Edition; Henderson, Sugden, \& Barnett, 2007). The MABC and its second edition, MABC-2, are the standard tests used worldwide for identifying developmental coordination disorder (DCD) (Blank, Smits-Engelsman, Polatajko, \& Wilson, 2012). In general, children with ASD during the ages of $6-15$ years showed a lower total standard score with this test, indicating that their general level of motor skill was significantly lower than that of typically developing children (Hilton et al., 2007; Whyatt \& Craig, 2012). However, an individual range of impairment has also been observed (Hirata et al., 2014). The question then arises about what factor determines the individual differences of motor skill impairments in children with ASD.

There is no dominant theory which explains the basis of motor skill impairments in children with DCD. The internal modeling deficit (IMD) was one of the hypotheses for explaining the cause of motor skill impairments. The IMD hypothesis was originally formulated following a series of experimental studies that explored cognitive characteristics of children with DCD (Katschmarsky et al., 2001; Wilson et al., 2004). According to this hypothesis, children with DCD have a deficit of their internal model of motor control. The internal model is a cognitive neuroscience concept of motor control and learning, and provides stability to the motor system by predicting the outcome of movements using the efference copy of motor command (Wolpert, 1997). This system is one of the essential parts of skilled movement, because the internal model allows subtle and rapid adjustments to the motor control before slow sensory-motor feedback becomes available. Thus, if one's internal model does not work well, some type of motor skill impairments will appear.

Many authors agree that motor imagery ability, which refers to the mental simulation ability of any motor act without motor execution, is reflective of one's ability to accurately form and manipulate internal models (Jeannerod, 1997; Skoura, Papaxanthis, Vinter, \& Pozzo, 2005). Previous studies have revealed that children with DCD show lower performance with such motor imagery tasks, especially using the mental rotation paradigm (Wilson et al., 2004; Williams et al., 2006; Williams, Thomas, Maruff, \& Wilson, 2008). The mental rotation task using limb-related stimuli was considered to reflect motor imagery ability (Parsons, 1987; Parsons, 1994; Conson et al., 2013). For example, participants were required to make a right-left decision about the presented hand stimulus at varying degrees from an upright position in the hand rotation task. Children with DCD generally reduce response accuracy in the hand rotation task, or display longer response time than typical children (Wilson et al., 2004), but not in the alphanumeric rotation task (Williams et al., 2006). Also, severities of motor skill impairments were interrelated with individual differences in hand rotation task performance in children with DCD (Williams et al., 2008). By these results, deficit of the internal model of motor control seems to be one possible cause of motor skill impairments in children with DCD. Unfortunately, few studies have conducted the mental rotation task using limb-related stimuli for children with ASD. To our knowledge, the relationship between motor skill impairments and motor imagery ability evaluated by the mental rotation paradigm in children with ASD has not been investigated. If the internal model also plays an important role in the motor skills of children with ASD, individual differences in motor skills must be strongly interrelated with the performance of mental rotation tasks using limb-related stimuli. A study of such issues may well provide further understanding and new insights into the underlying neural mechanism of motor skill impairments in children with ASD. The purpose of this study was to investigate the relationship between motor skill impairments and motor imagery ability in children with ASD. Hence, the MABC-2 and hand rotation task (Williams et al., 2006; Williams et al., 2008) were conducted.

\section{Methods}

\subsection{Participants}

Children with ASD from the elementary and junior high schools in the neighborhood of Tokyo Gakugei Univer- 
sity (Koganei City, Tokyo) who met the following conditions were recruited. First, children needed to be diagnosed by child psychiatrists as having Pervasive Developmental Disorder (PDD) based on the DSM-IV-TR criteria. Second, the children were confirmed to be free from severe sensory, neurological or muscular impairments such as blindness, low vision, deafness, and cerebral palsy. Third, to control for underlying cognitive effects such as difficulties in understanding and following instructions, the children were confirmed by their parents not to have severe intellectual difficulties. As a result, 19 children with ASD (18 males, 1 female) participated. Their chronological ages $(\mathrm{CAs})$ ranged from 7 to 16 years (mean $(\mathrm{M})=11.0$, standard deviation $(\mathrm{SD})=3.1)$. The participants' intelligence quotients (IQs) were measured by the Wechsler intelligence scale, Japanese version (Japanese WISC-4 Publication Committee, 2010), which is a standardized and commonly-used test in Japan. All 19 children were included in this study, because their full IQs ranged from 73 to 137 (mean $=95.7, \mathrm{SD}=14.5$ ). They did not appear to have additional intellectual difficulties. In this study, ASD included Asperger syndrome (AS, $n=3$ ), high-functioning autism (HFA, $n=3)$ and PDD-NOS $(n=13)$. However, we were unable to ascertain the concrete methods the child psychiatrists used for making diagnoses. To check the accuracy of ASD diagnoses, the Japanese version of Social Responsiveness Scale (SRS, Constantino \& Gruber, 2005; Kamio et al., 2013), the internationally accepted parent report measure of a child's social impairments in ordinary social settings was conducted with each participant's mother. The SRS is a 65-item questionnaire with each item was rated on a four-point scale, and the norm attached to the test manual is used when raw scores were converted to a total T-score $($ mean $=50, \mathrm{SD}=10)$. Higher scores indicate greater severity of social impairment. We classified participant's level of social impairment based on SRS scores according to the SRS manual. They ranged from the severe range $(76 \leq T, n=10)$, to the mild to moderate range $(60 \leq T \leq 75, n=9)$. Children in the "severe range" and "mild to moderate range" met the DSM criteria for PDD.

\subsection{Measures}

\subsubsection{Movement Assessment Battery for Children-2 (MABC-2)}

The Japanese version of the Movement Assessment Battery for Children, 2nd Edition (MABC-2) (Henderson et al., 2007), which we are now developing, was used to assess motor skill impairment. This test can assess children aged 3 - 16 years, and consists of 8 sub-tests which make up a total motor score and three sub-component scores: manual dexterity (MD), aiming and catching (AC), and balance (BAL). The structural validity of MABC-2 was confirmed using the confirmatory factor analysis (Schulz et al., 2011). The MD component includes three tasks: a one-hand pegboard task, a timed bimanual assembly task, and an untimed drawing task. The AC component includes tasks requiring the throwing of an object towards a target and the catching of an object by either one or both hands. The BAL component includes a static balance task and two dynamic balance tasks, which involve sustained, controlled movement (e.g., walking a line) and more explosive action (jumping or hopping). A norm attached to the test manual was used when raw scores were converted to four standard scores (mean $=10, \mathrm{SD}=3$ ) and percentiles. Lower standard scores indicate greater severity of motor skill impairment.

\subsubsection{The Hand Rotation Task (Williams et al., 2006; Williams et al., 2008)}

The Super Lab 4.5 (Cedrus Corporation, 2007) was used to present all stimuli for the hand rotation task, and to record the participant's response to the nearest $1 \mathrm{~ms}$. The hand stimuli were high-resolution images of a left or right hand in the back view, measuring $9 \mathrm{~cm}$ by $8 \mathrm{~cm}$. The fixation point cue was centered in the middle of a computer screen for $1 \mathrm{~s}$, and the hand stimuli were presented in $45^{\circ}$ increments between $0^{\circ}$ and $315^{\circ}$ when the fixation point cue disappeared. The participants sat in front of a computer screen with their hands resting on a response pad (RB-530, Cedrus Corporation, 2007) connected to the computer and covered by a soft towel to prevent participants from looking at their own hands. Prior to their hands being covered, participants located the appropriate response buttons on the response pad, left button for the left hand and right button for the right hand. Participants were instructed to determine whether the stimulus was a left hand or right hand as quickly and accurately as possible, and to respond by pressing the appropriate button on the response pad. They were not given any guidance on technique or strategy. When participants made a response, the next trial began. If participants did not respond within $10 \mathrm{~s}$, the stimulus disappeared and the next trial began. Examinations with all 16 trials $(8$ trials for left hand and 8 trials for right hand) were conducted 3 times in this study. All trials $\left(0^{\circ}, 45^{\circ}, 90^{\circ}, 135^{\circ}\right.$, $180^{\circ}, 225^{\circ}, 270^{\circ}$ and $315^{\circ}$ for each hand) were randomly presented to each participant. Participants completed four practice trials, followed by 16 test trials as 1 block. The remaining 2 blocks were examined while taking a 
break of approximately one minute after every block.

According to many previous studies using the hand rotation task (Wilson et al., 2004; Williams et al., 2006; Williams et al., 2008), the stimuli were combined for stimuli having the same angle of rotation, irrespective of the orientation, clockwise or anticlockwise direction (i.e., data for the $45^{\circ}$ and $315^{\circ}$ trials were combined). Thus, there were six trials at $0^{\circ}$ and $180^{\circ}$, while there were twelve trials at $45^{\circ}, 90^{\circ}$ and $135^{\circ}$. Mean correct response times (RTs) and response accuracies were calculated for each angle, and averaged over the detection of rotation for each participant. Anticipatory responses (less than $250 \mathrm{~ms}$ ) and abnormally delayed responses (greater than 2.5 times the mean RT for each angle for each participant) were excluded from analysis (Kosslyn et al., 1998). Each participant's mean correct RTs were plotted against the angle of rotation. A linear curve was fitted to the RT plot and a least squares method was used to obtain fit $\left(\mathrm{r}^{2}\right)$, slope and intercept. To ascertain the validity and reliability of this analysis, participant's mean correct RTs against each orientation were also plotted, whether in the clockwise $\left(0^{\circ}, 45^{\circ}, 90^{\circ}, 135^{\circ}\right.$ and $\left.180^{\circ}\right)$ or anticlockwise direction $\left(0^{\circ}, 315^{\circ}, 270^{\circ}, 225^{\circ}\right.$ and $\left.180^{\circ}\right)$. A signed rank test was conducted to analyze the differences of these two directions, revealing that the difference in direction was not significant for three measures, fit, slope, and intercept.

\subsection{Procedure}

Ethical approval for this study was obtained from the Research Ethics Board of Tokyo Gakugei University. Consent for participation in the study was obtained from the participant's parents, and the children were under no obligation to take part in the tests. Each participant was assessed in two sessions. The MABC-2 and hand rotation task were administered to all the children in the first session, and the Wechsler intelligence scale in the second session on another day. These sessions were conducted in a private room in Tokyo Gakugei University.

\subsection{Statistical Analysis}

The SPSS (ver. 12.0; SPSS Inc., 2004) was used for statistical analysis. All the measured values (see Table 1) were tested for deviation from normality. The tests revealed that not all the measures were normally distributed (i.e., AC and BAL scores of the MABC-2, all response accuracy (total, $0^{\circ}, 45^{\circ}, 90^{\circ}, 135^{\circ}$ and $180^{\circ}$ ), and intercept of the hand rotation task were not normally distributed). Thus, nonparametric analyses were used in this study. Spearman's rank correlation was used to analyze the relationship between the measures. In this study, we used a group with relatively heterogeneous CA and IQ ranges, and examined rank correlations between measured values and participant's CA or IQ. All the measured values were not significantly and strongly correlated with the participant's CA and IQ. Thus, participant's CA and IQ were not used as a covariate in all statistical analysis.

\section{Results}

\subsection{MABC-2}

Table 1 presents each measure of this study. According to many previous studies (Williams et al., 2006; Wil-

Table 1. Participant characteristics $(n=19)$.

\begin{tabular}{|c|c|c|c|}
\hline & Mean & Standard Deviation & Range \\
\hline \multicolumn{4}{|l|}{ MABC-2 Standard Scores } \\
\hline Total & 8.2 & 2.4 & $5-13$ \\
\hline Manual Dexterity (MD) & 7.5 & 2.7 & $3-13$ \\
\hline Aiming \& Catching (AC) & 8.2 & 2.4 & $2-12$ \\
\hline Balance (Bal) & 9.7 & 2.2 & $5-14$ \\
\hline \multicolumn{4}{|l|}{ Hand Rotation Task } \\
\hline Total Response Accuracy (\%) & 92.0 & 5.4 & $77.1-100.0$ \\
\hline Fit & 0.8 & 0.1 & $0.6-0.9$ \\
\hline Slope & 6.9 & 3.5 & $2.6-14.0$ \\
\hline Intercept & 1175.4 & 551.5 & $605.1-2512.9$ \\
\hline
\end{tabular}


liams et al., 2008), the 15th percentile of the MABC-2 total score was used as a cut-off point to identify motor difficulties. Forty-two percent of the children with ASD scored at least below the 15th percentile in their total standard score (below the 5th percentile, $\mathrm{n}=3$; 6th-15th percentile inclusive, $\mathrm{n}=5$ ). In the three sub-components, $47 \%$ of the children scored at least below the 15 th percentile in the MD (below the 5 th percentile, $n=4$; 6th-15th percentile inclusive, $n=5$ ), while $26 \%$ of them did so in the AC (below the 5th percentile, $n=2$; 6th15th percentile inclusive, $\mathrm{n}=5$ ); and $15 \%$ of them did so in the BAL (6th-15th percentile inclusive, $\mathrm{n}=3$ ).

\subsection{Hand Rotation Task}

\subsubsection{Response Accuracy}

Response accuracy showed the same tendency as previous studies (Williams et al., 2006; Williams et al., 2008); the greater the angle of rotation, the lower the response accuracy (Figure 1). The Friedman test was conducted to analyze the differences at each angle. The main effect of rotation angle was significant $\left(\chi^{2}(4)=17.6, p<0.01\right)$. However, there were no significant differences among the angles in the post hoc test (Dunn's test, $\alpha=0.05$ ).

\subsubsection{Fit and Slope}

To confirm the validity of the regression analysis, each participant's fit and slope coefficients were checked. All participants' fit coefficients were moderately high, i.e., they were higher by at least 0.6 . In this way, each regression estimate could be used as the participants' representative value of the hand rotation task. Next, all signs of slope coefficients were positive (see Table 1), meaning that the greater the angle of rotation (from $0^{\circ}$ to $180^{\circ}$ ), the longer the response time of all participants. This tendency was a typical example of mental rotation of hand stimulus and supports the claim that motor imagery was used to complete this task (Parsons 1994; Williams et al., 2008).

\subsection{Relationship between MABC-2 and Hand Rotation Task}

Spearman's rank correlations between each MABC-2 score and each regression estimate of the hand-rotation task were calculated. Table 2 shows the results. The MABC-2 total score was not significantly correlated with

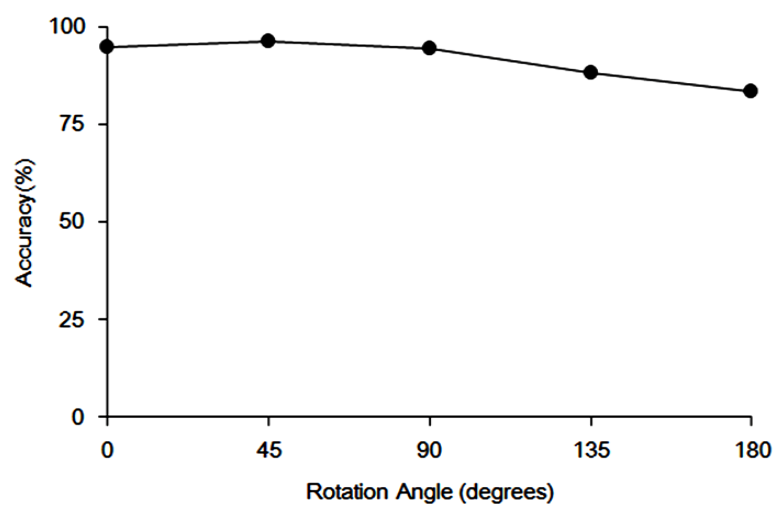

Figure 1. Mean response accuracy (\%) by rotation angle.

Table 2. Spearman's rank correlations between MABC-2 and hand rotation task.

\begin{tabular}{ccccc}
\hline & & Hand Rotation Task & \\
& Response Accuracy & Fit & Slope & Intercept \\
\hline MABC-2 & & & & \\
Total & -0.24 & 0.16 & -0.01 & -0.34 \\
MD & -0.02 & 0.35 & -0.23 & $-0.61^{* *}$ \\
AC & -0.33 & 0.11 & -0.25 & -0.02 \\
Bal & -0.25 & $-0.54^{*}$ & $0.57^{*}$ & 0.14 \\
\hline
\end{tabular}

${ }^{* *} p<0.01 ;{ }^{*} p<0.05$. 
all the regression estimates of the hand-rotation task. The MD score was significantly correlated negatively with the intercept of hand-rotation task, i.e., the lower the MD score, the higher the intercept. The AC score was not significantly correlated with the performance of hand-rotation task. The BAL score was significantly correlated positively with the slope of hand rotation task, i.e., the lower the BAL score, the lower the slope coefficient. The BAL score was also significantly correlated negatively with the fit coefficient, i.e., the lower the BAL score, the higher the linearity.

\section{Discussion}

This was the first study to examine the relationship between motor skills and motor imagery ability in children with ASD using the hand rotation task. Results indicated the possibility that severity of motor skill impairments was interrelated with motor imagery ability in children with ASD. First, fine motor control ability was strongly interrelated with the intercept of hand rotation task. Second, balance ability was interrelated with the fit and slope coefficients of the hand rotation task. From these results, several factors which affected the performance of MABC-2 were identified.

\subsection{Relationship between Fine Motor Control and Motor Imagery Ability}

For the moment, let us look closely at the relationship between fine motor control and motor imagery ability. Fine motor control ability evaluated by the MABC-2 was strongly interrelated with the general response speed of the hand rotation task (i.e., the intercept of hand rotation task). Previous studies revealed that severity of motor skill impairments was interrelated with individual differences in hand rotation task performance in children with DCD such as Williams et al. (2008). In the same study, the DCD severe (DCD-S) group responded more slowly and showed greater intercept than the DCD mild (DCD-M) group (these two groups were defined by the MABC total score). Relatively similar results were obtained from children with ASD in this study. This suggests the possibility that the internal modeling deficit plays an important role, both in children with DCD and children with ASD. However, the reason why only the MD score is interrelated with the intercept of hand rotation task is not yet clear. In the MD component of MABC-2, two of the three tasks measured and evaluated the time taken to accomplish a fine motor control task (i.e., shorter time was converted to a higher standard score). In the hand rotation task, mental rotations of the hand stimuli were very close to the corresponding real hand rotations (Parsons, 1987; Parsons, 1994). Thus, it seemed reasonable that the MD score of the MABC-2 was correlated with the response speed of the hand rotation task. In addition, Conson et al. (2013) recently reported that children with ASD did not differ with typical children in their performance of the letter rotation task, but showed a difference in their performances of the hand rotation task. These results indicate the possibility that slowness of the hand rotation task is not a reflection of the general cognitive processing speed. The relationship between the sub-components of MABC-2 and the performance of the hand rotation task has not been well investigated in children either with or without developmental disabilities. More research would be needed.

From a neurological perspective, comorbidity of motor skill impairments and social impairments indicates the possibility that there is a common brain dysfunction or cognitive deficit behind the lack of motor skill and social ability in children with ASD (Hilton et al., 2007; von Hofsten \& Rosander, 2012). The internal model deficit of the cerebellum seems to be one such factor. An "automatization" of movement execution is one of the essential factors of skilled movement (Shumway-Cook \& Woollacott, 2001), and the automatic process of social cognition or expression is also very important for guaranteeing smooth reciprocal interaction with others in everyday life (Romero-Munguía, 2008). In current neuroscience, it is considered that an internal model guarantees such automatic processes, and these internal models in various psychological domains are stored in the cerebellum (Ito, 2008). Cerebellar abnormality in autism has been consistently shown from neuroimaging studies (e.g., Courchensne, Hesselink, Jernigan, \& Yeung-Courchensne, 1987). For future research, investigation of the cerebellum's role in various psychological domains in children with ASD is warranted.

\subsection{Relationship between Balance and Motor Imagery Ability}

Turning now to the relationship between balance and motor imagery ability, Spearman's rank correlation revealed that the lower the BAL score was, the lower the slope coefficient would be. As Wilson et al. (2004) have 
already noted that there are two opposing views regarding the low value of the slope coefficient. First, a lower value slope coefficient reflects higher mental rotation ability. Second, a lower value slope coefficient reflects the possibility that the participant does not use the motor imagery effectively in the hand rotation task. Judging from the relationship with the balance ability, the latter view seems to be more appropriate. That is, in some ASD children not using motor imagery effectively, the hand rotation task becomes a choice reaction task irrespective of the rotation angle. This may contribute to better performance of the hand rotation task, such as a low value of the slope and good linearity. However, this style may contribute to poor balance ability, because the activation of motor imagery boosts positive effects for balance ability (Kawasaki \& Higuchi, 2013). The result of this study suggests the possibility that the inefficient use of motor imagery is sometimes convenient, such as during the hand rotation task, but is sometimes harmful, such as during the balance ability task. In children with DCD, some type of verbal instruction such as participants being asked to "imagine their own hand" is needed, to improve the performance of the hand rotation task (Williams et al., 2008). One must therefore check whether a similar result is observed in children with ASD.

\subsection{Limitations}

There are many methodological problems in this study. The small sample limits its generalizability. The lack of Japanese standardizations of the MABC-2 is an additional limitation. Moreover, the relationship between motor skill and motor imagery ability in typical children must be examined. Performance of the hand rotation task was not clearly interrelated with the AC score of MABC-2 in this study. The factors which contributed to the differences in AC scores should be investigated. Hirata et al. (2014) pointed out the possibility that several groups with different motor skill impairments might exist among children with ASD. The fact that each MABC-2 score was affected by different aspects of the hand rotation task suggested the possibility that several distinct motor imagery-related factors determined the characteristics of motor skill impairments in children with ASD. The relatively small sample size made a robust conclusion impossible. These are issues that can be addressed in future studies.

\section{Acknowledgements}

The authors would like to thank the generous participants who made this work possible. This study was supported by a Grant-in-Aid for Scientific Research $(245482,26780514)$ to Hirata from the Japan Society for the Promotion of Science (JSPS); a Grant-in-Aid for Scientific Research (25381301) to Nakai from the JSPS; and a Research Grant from the Ministry of Health, Labour, and Welfare, Japan to Nakai (H23 KOKORO-IPPAN-005). The research use in Japan and the development of the Japanese version of MABC-2 are based on a contract of Akio Nakai and Pearson Education Limited, with great support from Prof. Sheila E. Henderson, David A. Sugden, and Anna Barnett.

\section{References}

American Psychiatric Association (2000). Diagnostic and Statistical Manual of Mental Disorders (4th ed., Text Revision). Washington DC: American Psychiatric Association.

American Psychiatric Association (2013). Diagnostic and Statistical Manual of Mental Disorders (5th ed.). Arlington: American Psychiatric Association.

Blank, R., Smits-Engelsman, B., Polatajko, H., \& Wilson, P. (2012). European Academy for Childhood Disability (EACD): Recommendations on the Definition, Diagnosis and Intervention of Developmental Coordination Disorder (Long Version). Developmental Medicine \& Child Neurology, 54, 54-93. http://dx.doi.org/10.1111/j.1469-8749.2011.04171.x

Cedrus Corporation (2007). Super Lab 4.5. San Pedro: Cedrus Corporation.

Conson, M., Mazzarella, E., Frolli, A., Esposito, D., Marino, N., Trojano, L., Massagli, A., Gison, G., Aprea, N., \& Grossi, D. (2013). Motor Imagery in Asperger Syndrome: Testing Action Simulation by the Hand Laterality Task. PLoS ONE, 8 , e70734. http://dx.doi.org/10.1371/journal.pone.0070734

Constantino, J., \& Gruber, J. (2005). Social Responsiveness Scale (SRS) Manual. Los Angeles: Western Psychological Services.

Courchensne, E., Hesselink, J., Jernigan, T., \& Yeung-Courchensne, R. (1987). Abnormal Neuroanatomy in a Nonretarded Person with Autism: Unusual Findings with Magnetic Response Imaging. Archives of Neurology, 44, 335-341.

http://dx.doi.org/10.1001/archneur.1987.00520150073028

Henderson, S., \& Sugden, D. (1992). The Movement Assessment Battery for Children. London: The Psychological Corporation. 
Henderson, S., Sugden, D., \& Barnett, A. (2007). The Movement Assessment Battery for Children-2. Minnesota: Pearson Education, Inc.

Hilton, C., Wente, L., LaVesser, P., Ito, M., Reed, C., \& Herzberg, G. (2007). Relationship between Motor Skill Impairment and Severity in Children with Asperger's Syndrome. Research in Autism Spectrum Disorders, 1, 339-349. http://dx.doi.org/10.1016/j.rasd.2006.12.003

Hirata, S., Okuzumi, H., Kitajima, Y., Hosobuchi, T., Nakai, A., \& Kokubun, M. (2014). Relationship between Motor Skill and Social Impairment in Children with Autism Spectrum Disorders. International Journal of Developmental Disabilities, 60, 251-256. http://dx.doi.org/10.1179/2047387713Y.0000000033

Ito, M. (2008). Control of Mental Activities by Internal Models in the Cerebellum. Nature Reviews Neuroscience, 9, $304-313$. http://dx.doi.org/10.1038/nrn2332

Japanese WISC-4 Publication Committee (2010). Wechsler Intelligence Scale for Children (4th ed.). Tokyo: Nihon Bunka Kagakusha Co., Ltd.

Jeannerod, M. (1997). The Cognitive Neuroscience of Action. Oxford: Blackwell Publishers Ltd.

Kamio, Y., Inada, N., Moriwaki, A., Kuroda, M., Koyama, T., Tsujii, H. et al. (2013). Quantitative Autistic Traits Ascertained in a National Survey of 22529 Japanese School Children. Acta Psychiatrica Scandinavica, 128, 45-53. http://dx.doi.org/10.1111/acps.12034

Katschmarsky, S., Cairney, S., Maruff, P., Wilson, P., Tyler, P., \& Currie, J. (2001). The Ability to Execute Saccades on the Basis of Efference Copy: Impairments in Children with Developmental Coordination Disorder. Experimental Brain Research, 136, 73-78. http://dx.doi.org/10.1007/s002210000535

Kawasaki, T., \& Higuchi, T. (2013). Immediate Beneficial Effects of Mental Rotation Using Footstimuli on Upright Postural Stability in Healthy Participants. Rehabilitation Research and Practice, 2013, 1-7.

http://dx.doi.org/10.1155/2013/890962

Kosslyn, S., Digirolamo, G., Thompson, W., \& Alpert, N. (1998). Mental Rotation of Objects versus Hands: Neural Mechanisms Revealed by Positron Emission Tomography. Psychophysiology, 35, 151-161. http://dx.doi.org/10.1111/1469-8986.3520151

Parsons, L. M. (1987). Imagined Spatial Transformations of One's Hands and Feet. Cognitive Psychology, 19, $178-241$. http://dx.doi.org/10.1016/0010-0285(87)90011-9

Parsons, L. M. (1994). Temporal and Kinematic Properties of Motor Behavior Reflected in Mentally Simulated Action. Journal of Experimental Psychology: Human Perception and Performance, 20, 709-730. http://dx.doi.org/10.1037//0096-1523.20.4.709

Romero-Munguía, M. (2008). Mnesic Imbalance: A Cognitive Theory about Autism Spectrum Disorders. Annals of General Psychiatry, 7, 20. http://dx.doi.org/10.1186/1744-859X-7-20

Schulz, J., Henderson, S., Sugden, D., \& Barnett, A. (2011). Structural Validity of the Movement ABC-2 Test. Research in Developmental Disabilities, 32, 1361-1369. http://dx.doi.org/10.1016/j.ridd.2011.01.032

Shumway-Cook, A., \& Woollacott, M. H. (2001). Motor Control Second Edition. Maryland: Lippincott Williams \& Wilkins.

Skoura, X., Papaxanthis, C., Vinter, A., \& Pozzo, T. (2005). Mentally Represented Motor Actions in Normal Aging I. Age Effects on the Temporal Features of Overt and Covert Execution of Actions. Behavioural Brain Research, 165, $229-239$. http://dx.doi.org/10.1016/j.bbr.2005.07.023

SPSS Inc. (2004). SPSS 12.0 for Windows. Chicago: SPSS, Inc.

Von Hofsten, C., \& Rosander, K. (2012). Perception-Action in Children with ASD. Frontiers in Integrative Neuroscience, 6 , 1-6. http://dx.doi.org/10.3389/fnint.2012.00115

Whyatt, C., \& Craig, C. (2012). Motor Skills in Children Aged 7-10 Years, Diagnosed with Autism Spectrum Disorder. Journal of Autism and Developmental Disorders, 42, 1799-1809. http://dx.doi.org/10.1007/s10803-011-1421-8

Williams, J., Thomas, P. R., Maruff, P., \& Wilson, P. H. (2008). The Link between Motor Impairment Level and Motor Imagery Ability in Children with Developmental Coordination Disorder. Human Movement Science, 27, $270-285$. http://dx.doi.org/10.1016/j.humov.2008.02.008

Williams, J., Thomas, P., Maruff, P., Butson, M., \& Wilson, P. (2006). Motor, Visual and Egocentric Transformations in Children with Developmental Coordination Disorder. Child: Care, Health and Development, 32, 633-647. http://dx.doi.org/10.1111/j.1365-2214.2006.00688.x

Wilson, P. H., Maruff, P., Butson, M., Williams, J., Lum, J., \& Thomas, P. R. (2004). Internal Representation of Movement in Children with Developmental Coordination Disorder: A Mental Rotation Task. Developmental Medicine \& Child Neurology, 46, 754-759. http://dx.doi.org/10.1017/S001216220400129X

Wolpert, D. M. (1997). Computational Approaches to Motor Control. Trends in Cognitive Sciences, 1, 209-216. http://dx.doi.org/10.1016/S1364-6613(97)01070-X 